\title{
Thermal and viscosity properties of medium- and long-chain triacylglycerol blends
}

\begin{abstract}
The physical properties of medium- and long-chain triacylglycerol (MLCT) blends were characterized in this study. Blending MLCT oil with either palm olein or soybean oil changed the distribution of fatty acid groups, the crystallization and melting behavior of oil samples, and oil cloudiness. The crystallization and melting curves of both palm olein- and soybeanbased MLCT blends showed that the peaks shift to different transition temperatures depending on the percent of MLCT oil added. Viscosity analysis revealed that the oil viscosity was dependent on the temperature and triacylglycerol composition of the oil samples. Blending MLCT oil with either $50 \%$ palm olein or $50 \%$ soybean oil could assist in preventing crystal growth for the first $8 \mathrm{~h}$ of storage at 5 and $0 \mathrm{C}$, respectively.
\end{abstract}

Keyword: Medium- and long-chain triacylglycerols; Thermal behavior; Viscosity 